\title{
A FIELD-CIRCUIT APPROACH TO THE DESIGN ORIENTED EVALUATION OF THE NO-LOAD VOLTAGE HARMONICS OF SALIENT POLE SYNCHRONOUS GENERATORS
}
A. Di Gerlando
R Perini
I. Vistoli

Dipartimento di Elettrotecnica - Politecnico di Milano, Italy

\section{INTRODUCTION}

The Standards establish definite limits on the waveform distortion of the no-load voltage of the salient-pole synchronous generators, by means of the Total Harmonic Distortion (THD) and the Telephonic Harmonic Factor (THF): the constructional measures suited to reduce this distortion are numerous, and it is important to evaluate their effects during the design.

A method for the calculation of the no-load voltage distortion, taking into account the presence of the teeth, the winding and pole shoe characteristics, the saturation in all the magnetic branches and the skewing between stator and rotor is described and discussed.

An example of application is shown and the effect of some constructional measures is analysed.

\section{TEETH FLUXES AND E.M.F. HARMONICS}

The basic idea of the method is that the flux linkage of each winding turn equals the sum of the fluxes of the teeth included between the active sides of that turn.

Called $\gamma$ the electrical angle between two preestablished rotor and stator positions, let suppose to know all the teeth fluxes $\varphi_{\mathrm{tk}}(\mathrm{k}=1,2, \ldots \mathrm{c})$ (i.e. the vector $\left.\left[\varphi_{\mathrm{t}}\right]\right)$ for each value of $\gamma$. The flux linkage $\psi_{\mathrm{j}}$ of the $\mathrm{j}$-th coil of the considered phase winding is given by:

$$
\psi_{j}=N \cdot \sum_{j=1}^{c} a_{j k} \cdot \varphi_{t k}=N \cdot\left[a_{j}\right]^{T} \cdot\left[\varphi_{t}\right],
$$

where $a_{j k}$ equals +1 or 0 if the $j$-th coil (with $N$ turns) includes or not the k-th tooth.

The vector of the flux linkages of the $m$ series connected coils, belonging to the same phase winding, is:

$$
[\psi]=N \cdot[A] \cdot\left[\varphi_{t}\right]
$$

the $j$-th row of $[A]$ is the vector $\left[a_{j}\right]^{T}$ of the eq. (1).

Finally, the total flux linkage of each path of the phase winding is given by:

$$
\psi=\sum_{j=1}^{m} b_{j} \cdot \psi_{j}=[B]^{T} \cdot[\psi] ;
$$

[B] is the vector of the linkage signs: $b_{j}$ equals +1 (or 1) if the coil versor coincides (or does not) with that assumed positive for the teeth fluxes.

On the basis of eq.s (1)-(3), the flux linkage $\psi$ and the vector of the teeth fluxes $\left[\varphi_{t}\right]$ are linked as follows:

$$
\Psi=\mathrm{N} \cdot[\mathrm{B}]^{\mathrm{T}} \cdot[\mathrm{A}] \cdot\left[\varphi_{\mathrm{t}}\right]
$$

$[\mathrm{A}]$ and $[\mathrm{B}]$ include all the winding characteristics.

The knowledge of $\psi(\gamma)$ allows the direct calculation of the harmonics of the induced e.m.f. $e(t)$ :

$$
e(t)=-\frac{d \psi}{d t}=-\frac{d \psi}{d \gamma} \cdot \frac{d \gamma}{d t}=-\frac{d \psi}{d \gamma} \cdot \omega ;
$$

in fact, inserting eq. (6) in the Fourier integrals gives:

$$
\begin{aligned}
& E_{\text {mck }}=\frac{2}{T} \cdot \int_{0}^{T} c(t) \cdot \cos (k \cdot \omega \cdot t) \cdot d t=-\frac{\omega}{\pi} \cdot \int_{0}^{2 \pi} \frac{d \psi}{d \gamma} \cdot \cos (k \cdot \gamma) \cdot d \gamma, \\
& E_{\text {msk }}=\frac{2}{T} \cdot \int_{0}^{T}(t) \cdot \sin (k \cdot \omega \cdot t) \cdot d t=-\frac{\omega}{\pi} \cdot \int_{0}^{2 \pi} \frac{d \psi}{d \gamma} \cdot \sin (k \cdot \gamma) \cdot d \gamma .
\end{aligned}
$$

By integrating "per parts" the eq.s (7), one obtains:

$$
\begin{aligned}
& E_{m c k}=-k \cdot \frac{\omega}{\pi} \cdot \int_{0}^{2 \pi} \psi(\gamma) \cdot \sin (k \cdot \gamma) \cdot d \gamma, \\
& E_{m s k}=+k \cdot \frac{\omega}{\pi} \cdot \int_{0}^{2 \pi} \psi(\gamma) \cdot \cos (k \cdot \gamma) \cdot d \gamma .
\end{aligned}
$$

If requested, $\mathrm{e}(\mathrm{t})$ can be evaluated as follows:

$$
\Theta(t)=\sum_{k=1}^{k_{\max }}\left(E_{m c k} \cdot \cos (k \cdot \omega \cdot t)+E_{m s k} \cdot \sin (k \cdot \omega \cdot t)\right) \text {. }
$$

\section{MAGNETIC NETWORK DEFINITION}

The proposed method has the following features:

- it is applicable to any kind of winding;

- the solving matricial system is built easily and automatically, by means of the mesh analysis;

- the magnetic network is extended to the minimum necessary peripheral portion of the machine.

Called $\mathrm{p}_{\mathrm{g}}$ the number of poles in the cycle of the geometrical distribution of the active sides to the phases, if $\mathrm{p}_{\mathrm{g}}$ is even, the geometrical cycle is also an electromagnetic cycle; if $\mathrm{p}_{\mathrm{g}}$ is odd, the electromagnetic cycle includes two adjacent geometrical cycles (having opposite active side senses), with a number of poles $\mathrm{p}_{\mathrm{e}}=2 \cdot \mathrm{p}_{\mathrm{g}}$. Indicated with $n$ the number of slots/(phase-cycle), if $p_{g}$ is even the magnetic network is extended to $3 \cdot n$ slots (and teeth); if $\mathrm{pg}_{\mathrm{g}}$ is odd, it is extended to 6.n slots.

Fig.1 show's the magnetic network of a machine with $N^{\circ}$ of slots/(pole-phase) $q=n / p_{g}=7 / 4$ : the $N^{\circ}$ of slots of the network equals $3 \cdot n=21$; after the calculations, the assignment of the other fluxes is simply operated by considering the repetition of the electromagnetic cycle.

The network is composed as follows:

- constant reluctances (leakage between poles, $\theta_{d}$ );

- linear, position dependent reluctances (tooth reluctance at the air gap, between teeth and poles facing to them, indicated with $\left.\theta_{\mathrm{gk}}, \mathrm{k}=1,2, \ldots,\left(\mathrm{p}_{\mathrm{e}} / \mathrm{p}_{\mathrm{g}}\right) \cdot 3 \cdot \mathrm{n}\right)$;

- saturable reluctances (stator yoke reluctances between adjacent teeth $\left(\theta_{\text {sk }}\right)$, teeth $\left(\theta_{\mathrm{tk}}\right)$, rotor pole bodies 
$\left(\theta_{\mathrm{ph}}, \mathrm{h}=1,2, \ldots \mathrm{pe}\right)$, rotor yoke segments between adjacent poles $\left.\left(\theta_{\mathrm{rh}}\right)\right)$.

The number of the network magnetic meshes equals:

$$
\mathrm{N}_{\mathrm{r}}=\left(\mathrm{p}_{\mathrm{e}} / \mathrm{p}_{\mathrm{g}}\right) \cdot\left(3 \cdot \mathrm{n}+\mathrm{p}_{\mathrm{g}}\right)+1
$$

The assignment of the influencing pole of each tooth depends on the position of each tooth axis with respect to the rotor polar arcs; two cases must be considered:

- the permeance at the air gap of the tooth whose tooth pitch belongs totally to one rotor pole is evaluated on the basis of the unique corresponding flux tube;

- the tooth whose tooth pitch is crossed by an interpolar axis is modelled by a permeance connected to the nearest pole: its value is the difference between the two permeances of the flux tubes from the tooth itself and the two adjacent poles, Di Gerlando et al. (1).

The sequence of the serial numbering of the magnetic meshes is the following: at first the $\left(\mathrm{p}_{\mathrm{e}} / \mathrm{p}_{\mathrm{g}}\right) \cdot 3 \cdot \mathrm{n}$ slot meshes (1-21 in fig. 1); then the $\mathrm{p}_{\mathrm{e}}$ interpolar meshes (22-25 in fig.1); finally, the rotor yoke mesh (26).

The system of equations can be written as follows:

$$
[\theta] \cdot[\varphi]=[\mathbf{M}] \text {. }
$$

or, by making explicit the submatrices:

$$
\left[\begin{array}{cc}
{\left[\theta_{s}\right]} & {\left[\theta_{s r}\right]} \\
{\left[\theta_{s r}\right]^{T}} & {\left[\theta_{r}\right]}
\end{array}\right] \cdot\left[\begin{array}{l}
{\left[\varphi_{s}\right]} \\
{\left[\varphi_{r}\right]}
\end{array}\right]=\left[\begin{array}{l}
{\left[M_{s}\right]} \\
{\left[M_{r}\right]}
\end{array}\right] .
$$

$[\theta]$ is a symmetrical and dominant diagonal matrix.

The submatrix $\left[\theta_{\mathrm{S}}\right.$ ] (of the self and mutual stator slot reluctances) is squared and its order is $\left\{\left(p_{e} / p_{g}\right) \cdot 3 \cdot n\right\}$; each mesh corresponds to one slot and in each row of $\left[\theta_{S}\right]$ there are three non-zero terms only, because each slot has just two other slots nearby. $\left[\theta_{\mathrm{r}}\right]$ is squared too, and it includes the self and mutual rotor reluctances.

The submatrix $\left[\theta_{\mathrm{ST}}\right]$ of the mutual reluctances between stator and rotor has $\left\{\left(\mathrm{pe}_{\mathrm{e}} / \mathrm{p}_{\mathrm{g}}\right) \cdot 3 \cdot \mathrm{n}\right\}$ rows and $\left\{\mathrm{p}_{\mathrm{e}}+1\right\}$ columns and it is very sparse: the only elements different from zero are the interpolar leakage reluctances.

The vectors $\left[\varphi_{s}\right]$ (order $\left.\left\{\left(\mathrm{pe}_{\mathrm{e}} / \mathrm{p}_{\mathrm{g}}\right) \cdot 3 \cdot \mathrm{n}\right\}\right)$ and $\left[\varphi_{\mathrm{r}}\right]$ (order \{ $\left.\left.\mathrm{p}_{\mathrm{e}}+1\right\}\right)$ include the stator and rotor mesh fluxes. $\left[\mathrm{M}_{\mathrm{s}}\right]$ and $\left[\mathrm{M}_{\mathrm{r}}\right]$ include the stator and rotor mesh m.m.f.s and have the same order of the previous vectors (in the noload operation $\left[M_{S}\right]$ is identically zero).

For solving (11), an iterative procedure has been adopted, based on the gradual updating of the permeabilities of the saturable branches, (1).

An important aspect regards the number and the collocation of the rotor-stator positions necessary for the solution of the network: it can be shown that the best choice consists in adopting positions distant one from the other of the following mechanical rotation, (1):

$$
\Delta \beta=\frac{\delta}{2 \cdot a \cdot p_{g}}
$$

with $\delta$ mechanical angle between near teeth and "a" is:

$$
a=\text { integer }\left(h_{\max } / n\right)+1 \text {, }
$$

where $h_{\max }$ is the order of the harmonic e.m.f. with the higher order to be determined.

With this choice, the following results are obtained:

- thanks to the periodicity of the teeth-rotor configuration, the positions can be limited within one slot pitch $\left(\Delta \beta_{\text {tot }}=\delta\right.$ );

- evaluated the air gap permeance values of the tooth $N^{\circ} 1$ for all the positions distant $\Delta \beta$ within one half pole pitch, from these values one can obtain those of the other teeth, for each rotor position multiple of $\Delta \beta$;

- the choice of $\Delta \beta$ from (13), (14) allows to consider 12 points in the period of the $\mathrm{h}_{\text {max }}$-th harmonic e.m.f.

Thus, the network of fig.l must be solved $\left(2 \cdot a \cdot p_{g}\right)$ times, corresponding to the same number of equidistant rotor positions within one tooth pitch.

$\Theta_{81} \theta_{82} \Theta_{83} \Theta_{84} \theta_{85} \cdots$

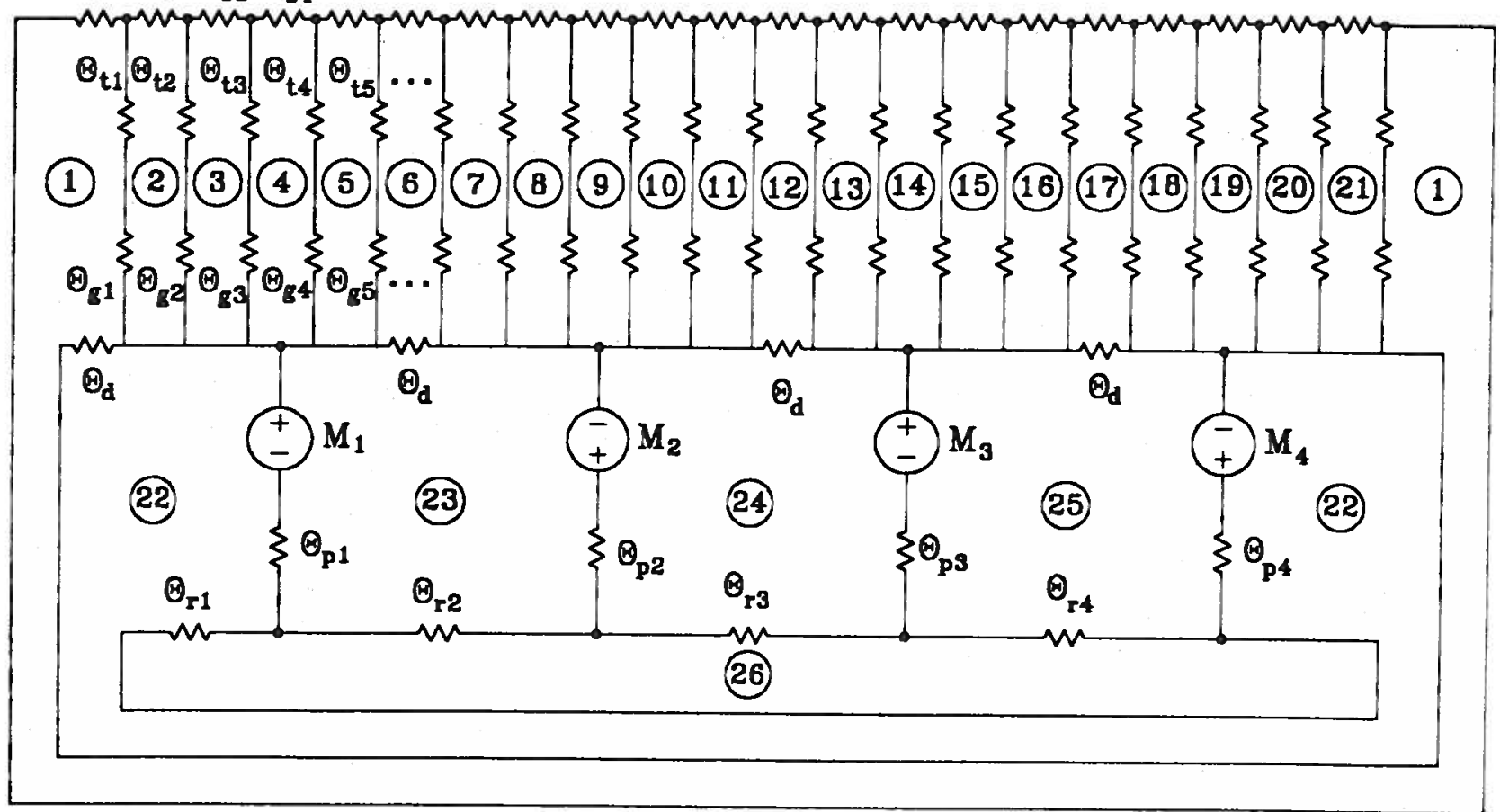

Fig.1 - Equivalent magnetic network of a salient pole alternator with $q=n / p_{g}=7 / 4$ : the network is extended to the number of slots of the geometrical cycle (in this case corresponding to the electromagnetic cycle). 


\section{EVAlUation OF THE PERMEANCES OF THE TEETH AT THE AIR GAP}

The parameter most difficult to be calculated is the tooth reluctance at the air gap, function of the distribution of the magnetic field at the air gap.

At present, one of the most used procedure for the solution of the magnetic field is the finite element method (FEM): it allows a high precision, but it is not simple and ready to be employed.

In the present paper an alternative method has been adopted, consisting of an approximate analytical procedure, based on a schematised description of the field lines and on the use of this well known integral:

$$
\lambda_{g}=\mu_{0} \cdot \int_{\tau_{t}} \frac{d \tau_{t}}{b_{g}}, \quad \text { where }
$$

- $\lambda_{g}$ is the specific tooth permeance at the air gap (i.e., the p.u. length permeance);

$-\tau_{t}$ is the tooth pitch (i.e., the p.u. length air gap area); - $b g$ is the length of the generic field line at the air gap within the considered tooth pitch.

Fig. 2 shows the map of a portion of the magnetic field obtained analysing an 8-pole alternator by the FEM: the real field lines and two polygonal lines approximating the field lines are shown.

The analytical procedure is based on the evaluation of the length $b_{g}$ of such approximating field lines, subdivided into two contributions, Di Gerlando et al., (2):

- line between the pole shoe and the internal stator circumference (length $\mathrm{b}_{\mathrm{gl}}$ );

- line between the stator circumference and the tooth side, modelled by circle arcs (length $b_{g_{2}}$ ).

In order to make this subdivision useful, the length $b_{p l}$ must be assumed independent on the presence of the teeth: in fact, the contraction of the field lines in front of the teeth is significant under the pole shoe only. where it is taken into account by the Carter's factor. As regards the calculation of $\mathrm{b}_{\mathrm{gl}}$, different polygonal lines are adopted for the intrapolar and interpolar zones: $A B C$ approximates an intrapolar field line. while DEFGH approximates an interpolar field line.

For a tooth totally included in the intrapolar zone, by solving eq.(15), a "closed form" for $\lambda_{\mathrm{g}}$ is obtained, (2):

$$
\lambda_{g}=\mu_{0} \cdot\left[\frac{b_{t}}{g_{0}}+2 \cdot \ln \left(1+\frac{b_{s}}{2 \cdot g_{0}}\right)\right] \text {, where: }
$$

- $g_{0}$ is the air gap along the tooth axis;

- $b_{t}$ and $b_{s}$ are the tooth and slot widths.

For the calculation of the specific permeance in the interpolar zone, a numerical integration of (15) must be performed: in any case, the subdivision into the two contributions of length $b_{g 1}$ and $b_{g 2}$ is useful, because it allows to determine all the necessary interpolar $b_{g 1}$ length values just at the beginning of the analysis.

Also the evaluation of the tooth permeance at the air gap of the teeth whose pitch is at the boundary between intrapolar and interpolar zones is made by numerically integrating eq. (15): $b_{g l}$ is given by polygonal lines, in part of the kind $A B C$ and in part of the kind DEFGH.

Of course, this approximate field model generates some errors, but they cause limited effects on the evaluation of the harmonics, (2).

As regards the extension to the case with relative skewing between stator and rotor, if the skewing is zero, the tooth permeance at the air gap equals:

$$
\Lambda_{\mathrm{g}}=\lambda_{\mathrm{g}} \cdot \ell \text {, }
$$

where $\ell$ is the machine axial length: in this case the magnetic network is extended to the machine length.

Vice versa, in case of skewing, it is necessary an axial subdivision in several segments, each represented by a different magnetic network, similar to that of fig. 1 .

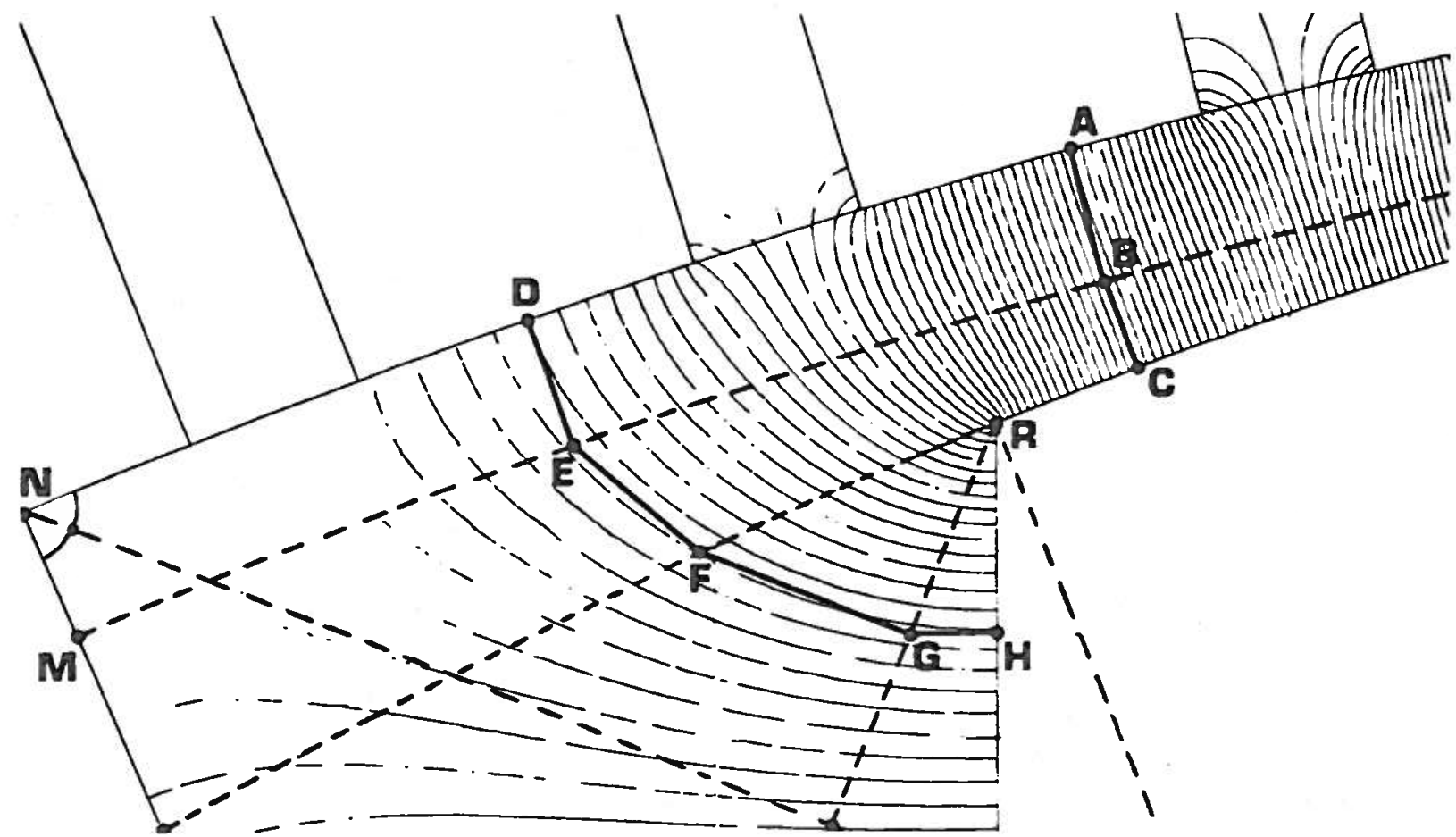

Fig. 2 - Map of the air gap magnetic field (intrapolar and interpolar zones) of a salient pole alternator with 8 poles (drawing obtained by the FEM): the approximating polygonal lines are shown too, employed in the analytical model. 
The solution of these networks for each position gives the flux contributions of the axial segments: the sum of these partial fluxes, performed individually for each tooth, gives the global fluxes, and then the waveforms.

For the above-mentioned subdivision it is useful that the angular shift among adjacent segments equals $\Delta \beta$ : this allows to limit the study within just one segment, obtaining the others with simple assignment criteria. In order to verify the approximation level connected with the use of eq.(15), a comparison with the FEM has been performed, for the machine data of Table 1 .

Table 1 - Example of constructional data for the calculation of the specific tooth permeances at the air gap.

\begin{tabular}{|l|l|}
\hline number of poles & $\mathrm{p}=8$ \\
\hline internal stator radius & $\mathrm{R}_{\mathrm{s}}=302 \mathrm{~mm}$ \\
\hline ratio min. air gap/intern. st. radius & $\mathrm{g}_{\mathrm{n}} / \mathrm{R}_{\mathrm{s}}=.0166$ \\
\hline ratio pole shoe radius/int. st. radius & $\mathrm{R}_{\mathrm{e}} / \mathrm{R}_{\mathrm{s}}=.715$ \\
\hline ratio pole shoe width/pole pitch & $\mathrm{b}_{\mathrm{e}} / \tau=.61 .7 .8$ \\
\hline ratio slot width/slot pitch & $\mathrm{b}_{\mathrm{s}} / \tau_{\mathrm{s}}=.3333$ \\
\hline ratio slot height/slot width & $\mathrm{h}_{\mathrm{s}} / \mathrm{b}_{\mathrm{s}}=5$ \\
\hline ratio pole shoe height/pole pitch & $\mathrm{h}_{\mathrm{e}} / \tau=.114 .177$ \\
\hline number of slots/(pole.phase) & $\mathrm{q}=4$ \\
\hline
\end{tabular}

Table 2 shows the specific permeance values, evaluated by the FEM and expressed in p.u., referred to the permeance of the tooth $\mathrm{N}^{\circ} 1$, at the centre of the pole.

The specific p.u. permeance of the tooth $\mathrm{N}^{\circ} 7$ refers to a half tooth ( $\left.7^{\prime}\right)$; in fact, the total tooth flux is zero, being the tooth $\mathrm{N}^{\circ} 7$ aligned with the interpolar axis.

Table 2 - Values of the specific tooth permeances at the air gap for the data of Table 1 (values in p.u., referred to $\left.\lambda_{\mathrm{gl}}\right): \quad \dot{\lambda}_{\mathrm{gk}}=\lambda_{\mathrm{gk}} / \lambda_{\mathrm{gl}}, \quad \lambda_{\mathrm{gl}}=4.580 \mu \mathrm{H} / \mathrm{m}$.

\begin{tabular}{|c|c||c|c|c|c|c|c|}
\hline$\frac{\mathrm{b}_{\mathrm{e}}}{\tau}$ & $\frac{\mathrm{h}_{\mathrm{e}}}{\tau}$ & $\dot{\lambda}_{\mathrm{g} 2}$ & $\dot{\lambda}_{\mathrm{g} 3}$ & $\dot{\lambda}_{\mathrm{g} 4}$ & $\dot{\lambda}_{\mathrm{g} 5}$ & $\dot{\lambda}_{\mathrm{g} 6}$ & $\dot{\lambda}_{\mathrm{g} \tau}$ \\
\hline \hline .8 & .177 & 0.958 & 0.850 & 0.716 & 0.583 & 0.339 & 0.036 \\
\hline .8 & .114 & 0.958 & 0.850 & 0.716 & 0.583 & 0.329 & 0.032 \\
\hline .7 & .177 & 0.958 & 0.850 & 0.716 & 0.534 & 0.185 & 0.018 \\
\hline .7 & .114 & 0.958 & 0.850 & 0.716 & 0.532 & 0.172 & 0.016 \\
\hline .6 & 1.177 & 0.958 & 0.850 & 0.708 & 0.349 & 0.096 & 0.010 \\
\hline .6 & .114 & 0.958 & 0.850 & 0.708 & 0.342 & 0.087 & 0.009 \\
\hline
\end{tabular}

The results of Table 2 suggest the following remarks:

- $\dot{\lambda}_{\mathrm{g} 2}$ and $\dot{\lambda}_{\mathrm{g} 3}$ are not affected by the extension and by the height of the pole shoe;

- ig4 is independent on the pole shoe height and it is weakly affected by its peripheral extension;

- for the teeth $N^{\circ} 5$ and 6 , there is a significant influence of the pole shoe extension;

- the half tooth permeance $\dot{\lambda}_{g} \tau$, very low, is greatly affected by the pole shoe extension and by its height.

Some ratio values between the permeances evaluated analytically $\left(\lambda_{\mathrm{gA}}\right)$ and by the FEM $\left(\lambda_{\mathrm{gF}}\right)$ are given:

1.tooth $\mathrm{N}^{\circ} 2$ (intrapolar zone); high pole shoe, with medium extension $\left(h_{e} / \tau=0.177 ; b_{e} / \tau=0.7\right)$ :

$$
\lambda_{\mathrm{g} 2 \mathrm{~A}} / \lambda_{\mathrm{g} 2 \mathrm{~F}}=1.00034
$$

2.tooth $\mathrm{N}^{\circ} 5$ (intrapolar zone); high pole shoe, with long extension $\left(\mathrm{h}_{\mathrm{e}} / \tau=0.177 ; \mathrm{b}_{\mathrm{e}} / \tau=0.8\right)$ :

$$
\lambda_{\mathrm{g} 5 \mathrm{~A}} / \lambda_{\mathrm{gSF}}=1.0075 \text {; }
$$

3.tooth $\mathrm{N}^{\circ} 6$ (interpolar zone); high pole shoe, with medium extension $\left(h_{e} / \tau=0.177 ; b_{e} / \tau=0.7\right)$;

$$
\lambda_{\mathrm{g} 6 \mathrm{~A} /} / \lambda_{\mathrm{g} 6 \mathrm{~F}_{\left(\mathrm{h}_{\mathrm{e}} \text { high }\right)}}=0.9238 \text {; }
$$

4.tooth $N^{\circ} 6$ (interpolar zone); low pole shoe, with medium extension $\left(h_{e} / \tau=0.114 ; b_{e} / \tau=0.7\right)$;

$$
\lambda_{\mathrm{g} 6 \mathrm{~A}} / \lambda_{\mathrm{g} 6 \mathrm{~F}_{\left(\mathrm{b}_{\mathrm{e}} \text { low }\right)}}=0.9908 \text {. }
$$

The first two examples show that the level of precision obtainable for teeth in intrapolar zone is satisfying.

The third ratio (interpolar zone) shows a higher error, nevertheless, it should be noted that this ratio refers to a high pole shoe $\left(h_{e} / \tau=0.177\right)$; if the same value of $\lambda_{g 6 \mathrm{~A}}$ is referred ( $4^{\circ}$ example) to the value corresponding to a low pole shoe $\left(\mathrm{h}_{\boldsymbol{e}} / \tau=0.114\right.$, ratio nearer to the values usually employed) the error in the permeance value is significantly smaller. The results of the cases 3 . and 4 . are different because the analytical model does not take into account the pole shoe height.

To sum up, we can conclude that the approximate calculation of the air gap tooth permeances is satisfactory.

\section{EXAMPLE OF CALCULATION}

Some calculation results for a synchronous machine with the main data of Table 3 are shown and discussed.

Table 3 - Main data of the illustrative alternator.

\begin{tabular}{|l|c|}
\hline Nominal power $\quad A_{n}[\mathrm{kVA}]$ & 7300 \\
\hline nominal voltage $\quad \mathrm{V}_{n}[\mathrm{~V}]$ & 6000 \\
\hline nominal frequency $\mathrm{f}_{\mathrm{n}}[\mathrm{Hz}] ;$ number of poles & $50 ; 6$ \\
\hline number of slots/(pole.phase) $\mathrm{q}$ & $13 / 2$ \\
\hline ratio (pole shoe width/pole pitch) & 0.7 \\
\hline ratio (max. air gap/min. air gap) & 1.5 \\
\hline
\end{tabular}

The diagrams of fig. 3 show (curves a) the waveforms of the phase e.m.f. $\left(V_{s}\right)$ and of the line-to-line e.m.f. $\left(V_{c}\right)$, and (histograms $b$ ) the corresponding RMS amplitude harmonic spectra (together with the fundamental RMS value $V_{1}$ ); the THD and THF values are given too.

The analysis refers to 3 distinct constructional choices:

- "full coil pitch" winding (pitch $y_{c} \approx 3 \cdot q \approx 19$ ); absence of rotor-stator skewing (diagrams a l, bl);

- "shortened coil pitch" winding (pitch $y_{c} \approx 2.5 \cdot q \approx$ 16); absence of skewing (diagrams a2, b2);

- "shortened pitch" winding (pitch $y_{c}=16$ ); stator-rotor skewing of one slot pitch (diagrams a3, b3).

One can conclude what follows:

- the diagrams al and bl show both the effect of the lower order harmonics (harmonic order in the range 5-31) and of the tooth harmonics (order 77 and 79);

- the "pitch shortening" greatly reduces the lower order harmonics, while the tooth harmonics remain high; also the amplitude of the fundamental decreases, but there is a waveform improvement, and a corresponding decrease of the coefficients THF and THD; 

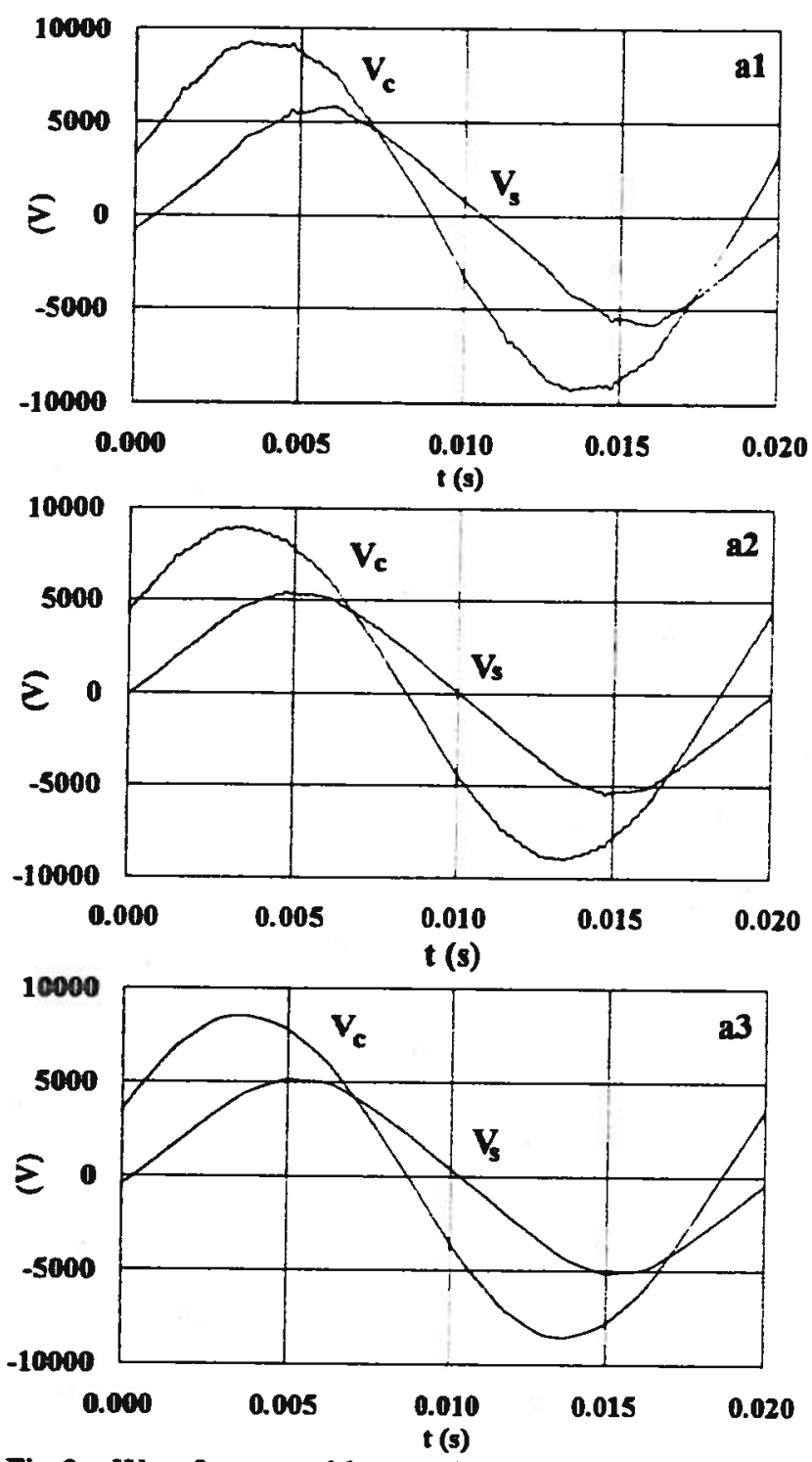
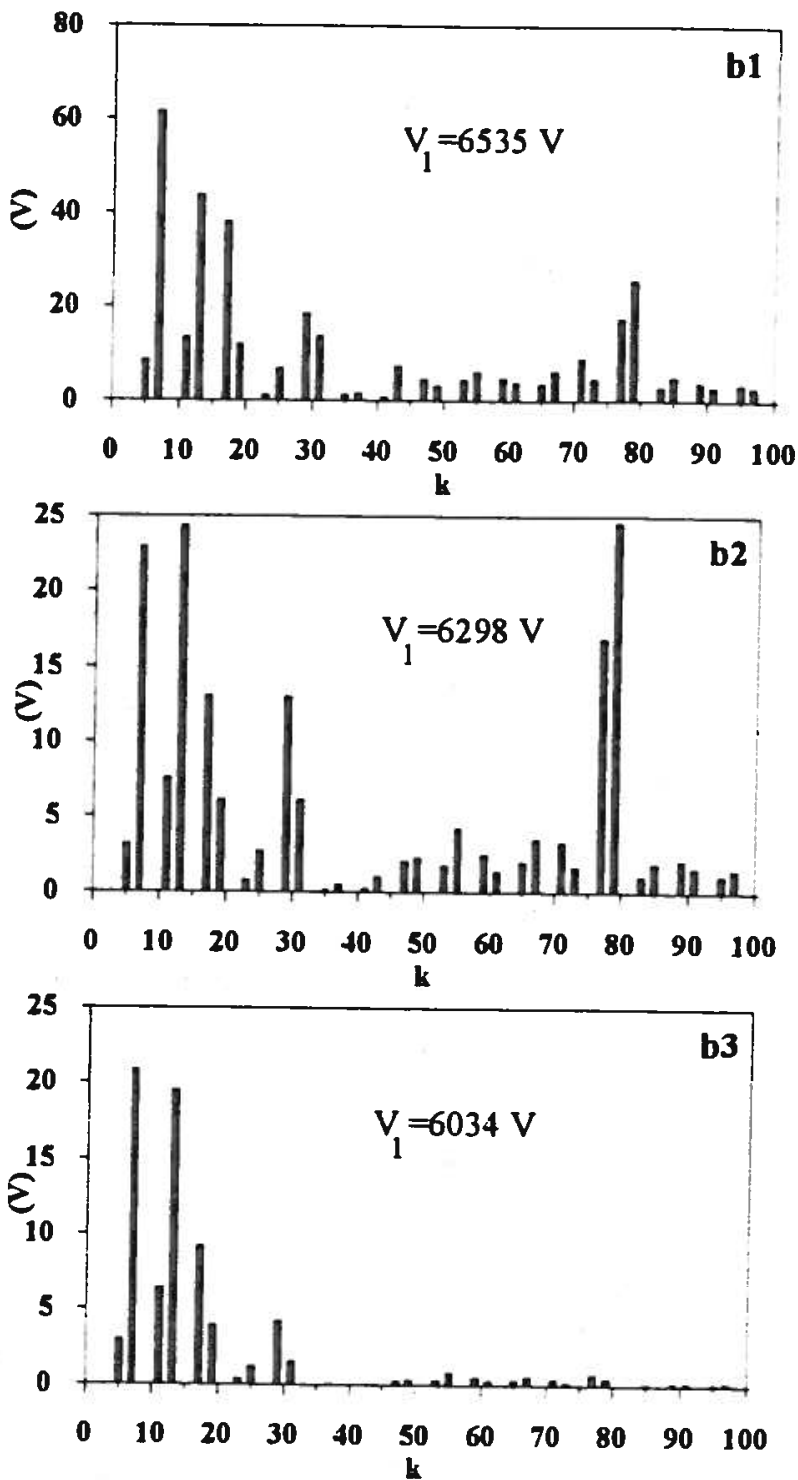

Fig.3 - Waveforms and harmonic spectra of the no-load voltage of the machine of Table 3:

curves $a=$ waveform of the phase voltage $\left(V_{S}\right)$ and of the line-to-line voltage $\left(V_{c}\right)$;

curves $\mathbf{b}=$ amplitude spectrum of the line-to line voltage (RMS values); $\mathrm{V}_{1}=\mathrm{RMS}$ value of the fundamental;

diagrams al, bl: coil pitch $y_{c}=19$; absence of stator-rotor skewing: $\quad T H F=1.25 \% ; \quad T H D=1.49 \%$

diagrams a2, b2: coil pitch $y_{c}=16$; absence of stator-rotor skewing: $\quad \mathrm{THF}=0.76 \% ; \quad \mathrm{THD}=0.81 \%$

diagrams a3, b3: coil pitch $\mathrm{y}_{c}=16$; skewing equal to one slot pitch: $\quad \mathrm{THF}=0.33 \% ; \quad \mathrm{THD}=0.52 \%$

- the skewing of one slot pitch between slots and rotor poles practically cancels the tooth harmonics: there is a further improvement of the waveform and a corresponding decrease of the THF and THD values.

These results, in good accordance with what can be expected on the basis of the classical theory, confirm the soundness of the described methodology.

\section{CONCLUSIONS}

The present paper has described a method for the calculation of the harmonic distortion of the no-load voltage of salient pole alternators: it takes into account the winding features, the shape of the pole shoes, the presence of the slots and the saturation of the ferromagnetic branches.

The method automatically assembles an equivalent magnetic network and performs an analytical approximate evaluation of the air gap permeances; this analyti- $\mathrm{cal}$ evaluation has given results very near to those of the finite element method.

An example has shown the potentialities and the usefulness of this calculation procedure during the design: in fact, it allows to foresee quickly and efficiently the effect of the design choices on the harmonic amplitudes and on the value of the THD and THF coefficients.

\section{REFERENCES}

1. A. Di Gerlando, R Perini, I. Vistoli, 1994, "Evaluation of No-Load Voltage Harmonics of Salient-Pole A.C. Generators: Analytical-Numerical Methodologies", VI IEEE-ICHPS Proc., 104-110.

2. A. Di Gerlando, R Perini, I. Vistoli, 1994. "Analytical Modelling of the Field at the Air-Gap of Salient-Pole A.C. Generators for the Estimation of the No-Load Voltage Harmonics", ibid., 97-103. 